\title{
A Case of Mitochondrial Trifunctional Protein Deficiency with $H A D H B$ Variants Diagnosed Using Whole-Exome Sequencing
}

\author{
Chan Kim, MD, Dajeong Lee, MD, Jiwon Lee, MD, Sung Yoon Cho, MD, Jeehun Lee, MD \\ Department of Pediatrics, Samsung Medical Center, Sungkyunkwan University School of Medicine, Seoul, Korea
}

Received: June 14, 2021

Revised: July 20, 2021

Accepted: July 23, 2021

Corresponding author:

Jiwon Lee, MD

Department of Pediatrics, Samsung

Medical Center, Sungkyunkwan

University School of Medicine, 81

Irwon-ro, Gangnam-gu, Seoul

06351, Korea

Tel: +82-2-3410-0811

Fax: +82-2-3410-0043

E-mail: jwjw.lee@samsung.com
Mitochondrial trifunctional protein (MTP) is a hetero-multimeric enzyme complex comprised of four $\alpha$-subunits and four $\beta$-subunits that is involved in mitochondrial $\beta$-oxidation of fatty acids. MTP deficiency occurs due to variations in the HADHA or $H A D H B$ genes, which are located on chromosome 2 p23 and encode the $\alpha$-subunits and $\beta$-subunits of MTP, respectively. The symptoms of MTP deficiency vary according to the degree of metabolic derangement, ranging from mild sensorimotor polyneuropathy with episodic rhabdomyolysis to severe infantile cardiomyopathy [1]. Because MTP deficiency caused by variants of the $H A D H B$ gene has heterogeneous manifestations at both the molecular and phenotypic levels, it is sometimes difficult to confirm the diagnosis through metabolic tests. Next-generation sequencing (NGS)-based genetic studies can provide a definite diagnosis when metabolic tests are not definite [2-4]. We report the case of a patient who presented with recurrent rhabdomyolysis accompanying viral infections and was diagnosed with MTP deficiency attributed to variants of the $H A D H B$ gene identified using whole-exome sequencing (WES).

A 21-month-old female patient presented to the hospital with abdominal pain, vomiting, and generalized weakness. The patient was born fullterm with no perinatal problems and showed normal development. She was the second baby in a pair of dichorionic, diamniotic twins. Blood tests revealed elevated levels of liver enzymes, troponin I, creatine kinase (CK), and CKMB isoenzyme (Table 1). Because the Mycoplasma pneumoniae antibody titer was elevated (1:1,280), mycoplasma infection was suspected. Liver enzymes, cardiac enzymes, and CK levels decreased on the third day of hospitalization with conservative management, and the initial symptoms disappeared after 5 days. Metabolic tests, including urine organic acid and plasma amino acid levels, did not indicate specific metabolic disorders. A plasma acylcarnitine test revealed elevated C14$\mathrm{OH}, \mathrm{C} 16-\mathrm{OH}, \mathrm{C} 18: 1-\mathrm{OH}$, and C18-OH levels, which led to clinical suspicion of MTP deficiency.

One month after the previous admission, the patient presented to the pediatric emergency room with a persistent fever up to $40^{\circ} \mathrm{C}$ and poor oral intake. She had generalized weakness and lay in bed most of the time. Her voice was quiet, and she frequently choked while eating.

A physical examination revealed pharyngeal injection and mild tenderness in both lower legs. The patient was alert and showed a normal cra-

Copyright (C) 2021 Korean Child Neurology Society

This is an Open Access article distributed under the terms of the Creative Commons Attribution Non-Commercial License (http://creativecommons.org/licenses/by-nc/4.0/) which permits unrestricted non-commercial use, distribution, and reproduction in any medium, provided the original work is properly cited. 


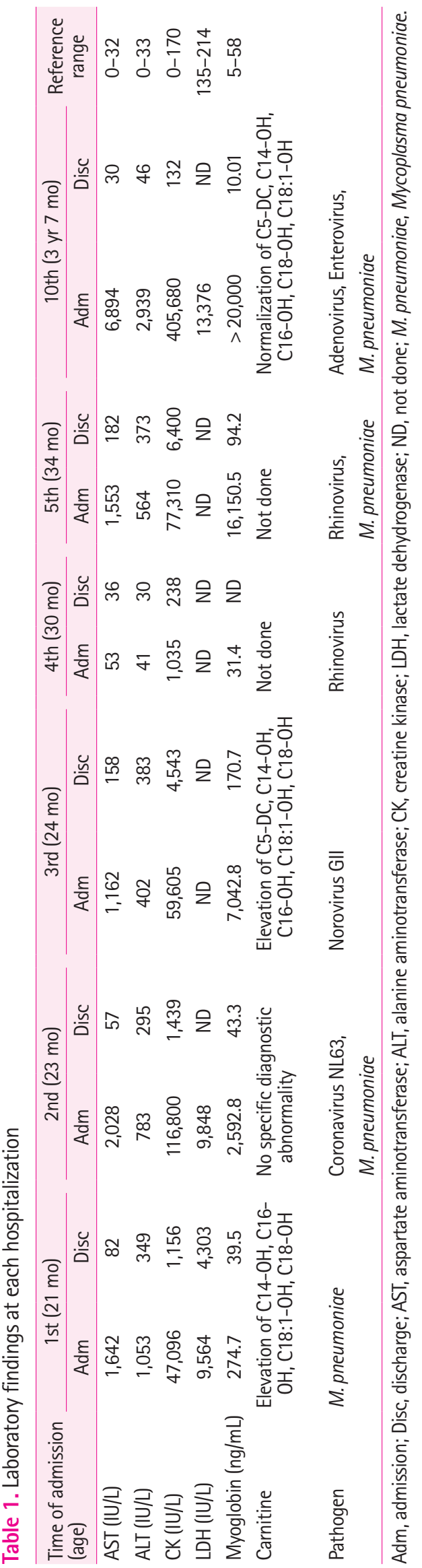

nial nerve examination. On motor examination, the motor power of the lower extremities was found to be mildly decreased on both sides (grades 4 to 5). The deep tendon reflexes of the knee and ankle were $1+$ on both sides. Laboratory findings showed elevated aspartate aminotransferase (AST), alanine aminotransferase (ALT), CK, myoglobin, and lactate dehydrogenase (LDH) levels (Table 1). Urinalysis revealed myoglobinuria. Infection with human coronavirus NL63 was confirmed using respiratory virus polymerase chain reaction (PCR). The previously elevated blood test levels (AST, ALT, CK, myoglobin, and LDH) decreased in the follow-up tests with intravenous hydration (Table 1). Along with this, the patient's activity gradually improved. As the previous plasma acylcarnitine test had revealed elevated C14-OH, C16-OH, $\mathrm{C} 18: 1-\mathrm{OH}$, and $\mathrm{C} 18-\mathrm{OH}$ levels, urine organic acid and plasma acylcarnitine levels were checked again to reach a definite diagnosis. However, these tests did not enable a specific diagnosis.

At the age of 24 months, the patient was readmitted to the hospital with vomiting, poor oral intake, general weakness, and red-colored urine. A stool PCR test detected norovirus. The patient had elevated liver enzyme and CK levels, which markedly improved after 5 days (Table 1). Although metabolic myopathy was strongly suspected clinically, the repeated metabolic tests were inconclusive, and WES was performed. Informed consent was obtained from the patient and her parents. This study was approved by the Institutional Review Board of the Samsung Seoul Hospital (IRB No. 2020-04-197). Compound heterozygous $H A D H B$ variants (NM_000183.3, c.340A > G;p.N114D and c.919A > G;p.N307D) were detected in WES. Sanger sequencing revealed that each parent had one of these variants. Two variants of the $H A D H B$ gene are located in the thiolase-N domain of the $\beta$-subunits of MTP, which harbor long-chain 3-ketoacyl-CoA thiolase activity [5]. These variants have an occurrence of $<1 \%$ in reference databases, such as the Korean Reference Genome Database and Genome Aggregation Database. In silico prediction tools predicted the detected missense changes to be damaging. Detailed data on the effect prediction and conservation of the variants are described in Table 2. The patient was diagnosed with MTP deficiency due to likely pathogenic variants of the $H A D H B$ gene.

The patient had repeated episodes of dehydration and rhabdomyolysis that seemed to mirror those of hand-foot-mouth disease, acute gastroenteritis, acute upper respiratory infection, and acute myocarditis (fourth, fifth, eighth, and 10th admissions, respectively) (Table 1). The symptoms and severity were slightly different at each admission; however, rhabdomyolysis and motor weakness were common findings. In addition to providing supportive care during each admission, long-term treatment for MTP deficiency was started at the age of 3 with a low-fat and high-carbohydrate 
Table 2. Effect prediction of the identified variants

\begin{tabular}{llll}
\hline & Prediction tool & \multicolumn{1}{c}{ c.340A>G;p.N114D } & \multicolumn{1}{c}{ c.919A > G;p.N307D } \\
\hline Minor allele frequency & gnomAD & $0.01088 \%(2 / 18,390$ alleles in East Asian) & 0.01087 (2/18,394 alleles in East Asian) \\
& KRGDB & 0.0009 & 0.0003 \\
In silico & SIF & Deleterious (0) & Deleterious (0) \\
& PolyPhen-2 & Probably damaging (0.996) & Probably damaging (0.959) \\
& REVEL & Deleterious (0.894) & Deleterious (0.919) \\
Conservation & GERP++_RS & 5.24 & 5.43 \\
Domain & InterPro & Thiolase_N & Thiolase_N \\
\hline
\end{tabular}

diet. Fat-soluble vitamins and L-carnitine were supplemented with avoidance of fasting and infection.

At the last follow-up at age 6, the patient showed normal growth, good activity, and tolerable blood laboratory tests (AST, $30 \mathrm{IU} / \mathrm{L}$; ALT, $21 \mathrm{IU} / \mathrm{L}$; CK and myoglobin, $198 \mathrm{IU} / \mathrm{L}$ and $26.9 \mathrm{ng} / \mathrm{mL}$, respectively).

The recurrent elevation in liver enzymes, $\mathrm{CK}$, and myoglobin levels coupled with motor weakness could be a clue for the diagnosis of MTP deficiency. Fu et al. [6] reported a case of an 8-year-old Chinese girl with progressive lower limb weakness and lethargy, which was aggravated after fever; this clinical feature was similar to that of our patient. The patient described by Fu et al. [6] had intermittent increases in CK levels and peripheral nerve injury in a nerve conduction study. This supported the possibility of metabolic neuromyopathy. A homozygous missense $H A D H B$ variant was detected in the patient, and MTP deficiency was diagnosed [6]. In a report including 50 European patients with long-chain 3-hydroxyacyl-CoA dehydrogenase (LCHAD) deficiency, the patients also showed similar clinical and laboratory findings [7]. The age of symptom onset varied widely (range, 1 day to 26 months), but only seven patients (15\%) presented with symptoms during the neonatal period. Most patients $(n=43,86 \%)$ manifested chronic problems, such as hepatic dysfunction, failure to thrive, hypotonia, and feeding difficulties, rather than acute problems. In that study, 14 patients died before the diagnosis. Due to the nonspecific nature of the symptoms, their clinical significance tended to be missed in the early stages of the disease [7].

Three clinical phenotypes are associated with MTP or LCHAD deficiency: lethal (severe, neonatal onset), hepatic (moderate, infantile onset), and neuromyopathic (mild, late adolescent onset). It has been reported that patients with heterozygous missense $H A D H B$ variants present milder myopathic phenotypes $[4,8]$. Spiekerkoetter et al. [3] reported 11 patients with MTP deficiency showing the neuromyopathic phenotype. Eight patients harbored pathogenic variants of the $H A D H B$ gene, and six of them were found to have compound heterozygous missense variants [3]. In another report analyzing the molecular and phenotypic relationships of 15 patients with $H A D H B$ variants, 12 different missense variants, three nonsense variants, and one frameshift variant were found. Ten patients harbored missense variants, and seven of them (70\%) showed milder phenotypes and were diagnosed with later onset ( 1 to 6 years) MTP deficiency [4]. Therefore, it is possible that missense variants of the $H A D H B$ gene induce milder phenotypes than nonsense or frameshift variants. In our report, the presence of two compound heterozygous missense $H A D H B$ variants may have contributed to the relatively late onset and neuromyopathic phenotype of the disease.

The diagnosis of metabolic disorders begins with suspicion arising from clinical symptoms. Although recurrent rhabdomyolysis is a characteristic manifestation of MTP deficiency, it can be masked during early infancy. Our patient experienced repetitive neuromyopathy worsened by viral illness, but diagnosis was still delayed. In addition, metabolic tests- the traditional diagnostic method for MTP deficiency — can be affected by the symptoms or activity of diseases and may be inconclusive for a diagnosis [9]. As repeated metabolic tests did not confirm our suspicion of a metabolic disorder, we performed WES and made a diagnosis of MTP deficiency arising as a result of $H A D H B$ variants. NGS provides an alternative method for diagnosing metabolic myopathies. Diebold et al. [2] used a target gene panel containing more than 1,500 genes associated with known human diseases to detect disease-causing variants of $H A D H A$ or $H A D H B$ genes in a cohort of 403 patients. They highlighted the importance of genetic testing for disease diagnosis and the usefulness of phenotype-based NGS because of the clinically variable spectrum of metabolic diseases and difficulty in differentiating MTP deficiency from other fatty acid oxidation disorders, including carnitine palmitoyl-CoA transferase 1 and 2 deficiency, carnitine transporter deficiency, and multiple acyl-CoA dehydrogenase deficiency $[2,10]$. In addition, a Chinese patient was diagnosed using NGS-based gene panel testing comprising 153 genes for inherited metabolic diseases [6]. In our study, NGSbased WES played a decisive role in the confirmatory diagnosis of 


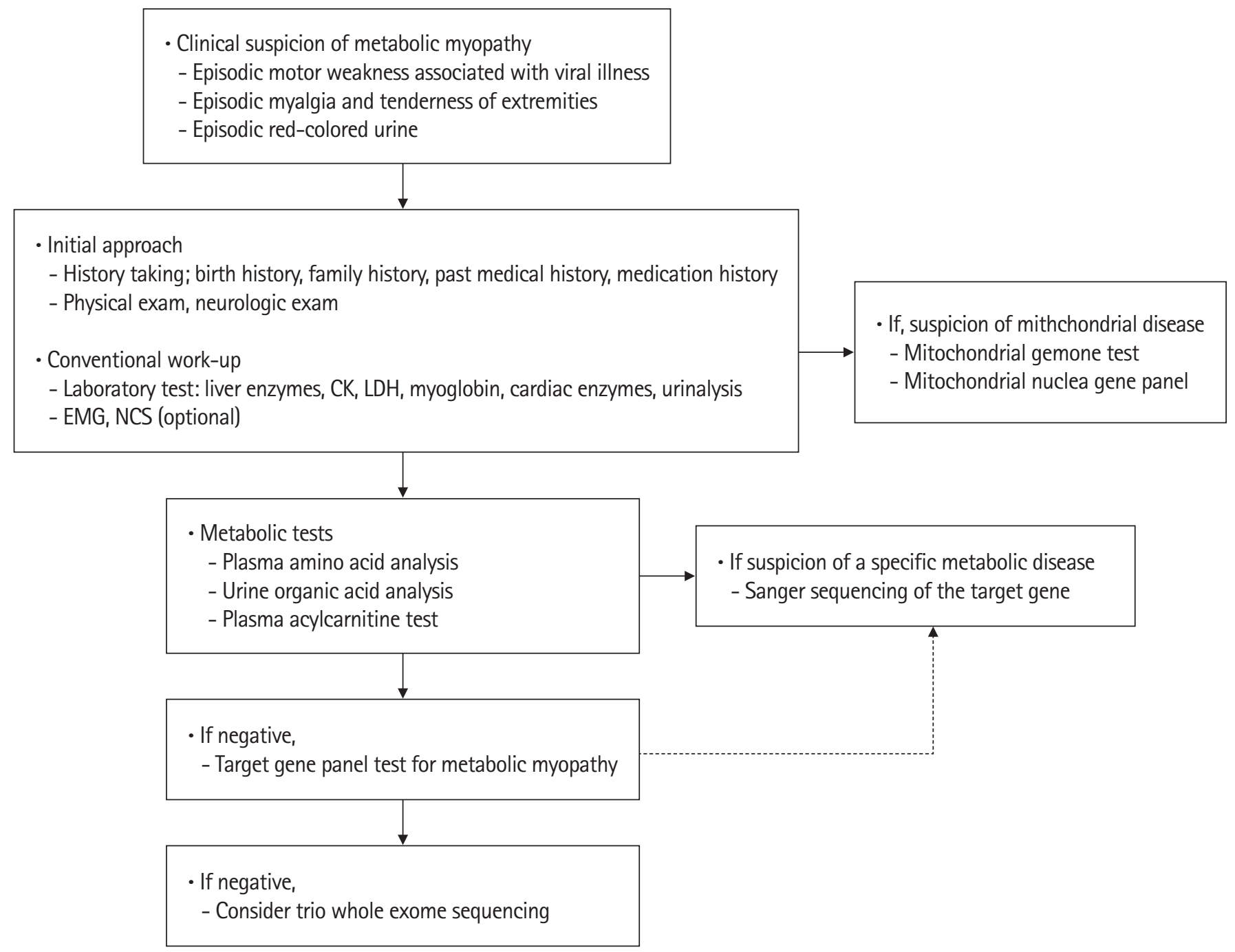

Fig. 1. Suggested diagnostic algorithm for the patients with suspected metabolic myopathy. CK, creatine kinase; LDH, lactate dehydrogenase; EMG, electromyogram; NCS, nerve conduction study.

a patient with suspected hereditary metabolic myopathy. We suggest a diagnostic algorithm for patients with suspected metabolic myopathy in Fig. 1. In conclusion, our patient exhibited a relatively late-onset and mild neuromyopathic phenotype with rhabdomyolysis. The results of metabolic tests were variable and inconclusive, despite repetitive testing, whereas WES provided a rapid and confirmatory diagnosis by identifying two compound heterozygous $H A D H B$ variants. Thus, NGS-based genetic testing could serve as a first-tier test for patients with suspected metabolic myopathy.

\section{Conflicts of interest}

No potential conflict of interest relevant to this article was reported.

\section{ORCID}

Chan Kim, https://orcid.org/0000-0003-3607-9737

Jiwon Lee, https://orcid.org/0000-0002-8456-684X

\section{Author contribution}

Conceptualization: JwL and JhL. Data curation: CK and DL. Formal analysis: JwL and SYC. Funding acquisition: JhL. Methodology: SYC and JhL. Project administration: JwL. Visualization: CK and DL. Writing-original draft: CK. Writing-review \& editing: JwL and JhL. 


\section{References}

1. Park HD, Kim SR, Ki CS, Lee SY, Chang YS, Jin DK, et al. Two novel $\mathrm{HADHB}$ gene mutations in a Korean patient with mitochondrial trifunctional protein deficiency. Ann Clin Lab Sci 2009;39:399-404.

2. Diebold I, Schon U, Horvath R, Schwartz O, Holinski-Feder E, Kolbel $\mathrm{H}$, et al. HADHA and HADHB gene associated phenotypes: identification of rare variants in a patient cohort by next generation sequencing. Mol Cell Probes 2019;44:14-20.

3. Spiekerkoetter U, Bennett MJ, Ben-Zeev B, Strauss AW, Tein I. Peripheral neuropathy, episodic myoglobinuria, and respiratory failure in deficiency of the mitochondrial trifunctional protein. Muscle Nerve 2004;29:66-72.

4. Spiekerkoetter U, Sun B, Khuchua Z, Bennett MJ, Strauss AW. Molecular and phenotypic heterogeneity in mitochondrial trifunctional protein deficiency due to beta-subunit mutations. Hum Mutat 2003;21:598-607.

5. Xia C, Fu Z, Battaile KP, Kim JP. Crystal structure of human mitochondrial trifunctional protein, a fatty acid $\beta$-oxidation me- tabolon. Proc Natl Acad Sci U S A 2019;116:6069-74.

6. Fu X, Zheng F, Zhang Y, Bao X, Wang S, Yang Y, et al. Mitochondrial trifunctional protein deficiency due to $\mathrm{HADHB}$ gene mutation in a Chinese family. Mol Genet Metab Rep 2015; 5:80-4.

7. den Boer ME, Wanders RJ, Morris AA, IJlst L, Heymans HS, Wijburg FA. Long-chain 3-hydroxyacyl-CoA dehydrogenase deficiency: clinical presentation and follow-up of 50 patients. Pediatrics 2002;109:99-104.

8. Shahrokhi M, Shafiei M, Galehdari H, Shariati G. Identification of a novel HADHB gene mutation in an Iranian patient with mitochondrial trifunctional protein deficiency. Arch Iran Med 2017;20:22-7.

9. Olpin SE, Clark S, Andresen BS, Bischoff C, Olsen RK, Gregersen $\mathrm{N}$, et al. Biochemical, clinical and molecular findings in $\mathrm{LCHAD}$ and general mitochondrial trifunctional protein deficiency. J Inherit Metab Dis 2005;28:533-44.

10. Gregersen N, Andresen BS, Pedersen CB, Olsen RK, Corydon TJ, Bross P. Mitochondrial fatty acid oxidation defects: remaining challenges. J Inherit Metab Dis 2008;31:643-57. 